\title{
Lymphocytosis with Smudge Cells Is Not Equivalent to Chronic Lymphocytic Leukemia
}

\author{
David Susman $^{a} \quad$ Russell Price $^{b} \quad$ Rouslan Kotchetkov ${ }^{c}$ \\ aDepartment of Medicine, Western University, London, ON, Canada; ${ }^{b}$ Department of \\ Pathology, Royal Victoria Regional Health Centre, Barrie, ON, Canada; 'Department of \\ Oncology, Royal Victoria Regional Health Centre, Barrie, ON, Canada
}

\section{Keywords}

Lymphocytosis · Smudge cells · Chronic lymphocytic leukemia · Flow cytometry · Lymphoproliferative disorder

\begin{abstract}
Chronic lymphocytic leukemia (CLL) often presents with lymphocytosis and smudge cells (SCs) on routine peripheral blood (PB) tests. In some cases, these findings are assumed to be sufficient to diagnose CLL. We present a 54-year-old male who was referred for further management of progressing CLL. At the initial presentation, he looked unwell and had diffuse lymphadenopathy and splenomegaly. Blood work showed normocytic anemia (hemoglobin $72 \mathrm{~g} / \mathrm{L}$ ), thrombocytopenia (platelet count $74 \times 10^{9} / \mathrm{L}$ ), leukocytosis (white blood cell count $135.5 \times$ $10^{9} / \mathrm{L}$ ) including lymphocytosis $\left(130.1 \times 10^{9} / \mathrm{L}\right)$, and the presence of SCs on a PB smear. Additional workup including flow cytometry ( $F C$ ), bone marrow biopsy, and lymph node biopsy led to a diagnosis of leukemic stage of advanced-stage mantle cell lymphoma. Although lymphocytosis with SCs is more frequently and in higher quantities seen in CLL they are not pathognomonic and can be present in a variety of lymphoproliferative disorders. Additional diagnostic examination of cell morphology and FC to assess clonality and determine the immunotype of lymphocytes are required to establish an accurate diagnosis and determine appropriate further management of the specific disease type.
\end{abstract}

(C) 2021 The Author(s).

Published by S. Karger AG, Basel

\section{Introduction}

Lymphocytosis refers to an increase of peripheral blood (PB) lymphocyte count >3.0-4.0 $\times$ $10^{9}$ cells/L in most laboratories. Lymphocytosis could be primary (malignant, monomorphic, and clonal) due to lymphoproliferative disorders (LPDs) or secondary (reactive, pleomorphic, 

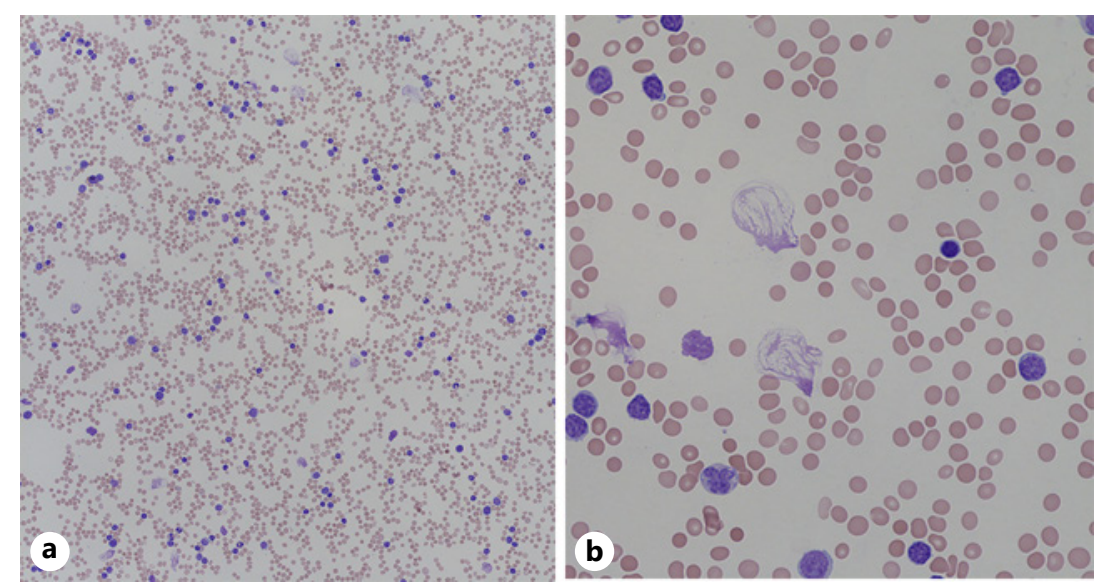

Fig. 1. PB smear. a Lymphocytosis in PB with small to intermediate size mature lymphocytes; original magnification, $\times 200$. $\mathbf{b}$ multiple SCs, original magnification, $\times 400$. SC, smudge cell; PB, peripheral blood.

and nonclonal). Chronic Lymphocytic Leukemia (CLL) is the most common form of adult leukemia in Western countries. The median age at diagnosis is approximately 70 years [1]. It frequently presents with incidental findings of lymphocytosis on routine blood work and is often accompanied by the presence of smudge cells (SCs) on a PB smear. Similar findings can result from other benign or malignant conditions that can mimic CLL.

\section{Case Report/Case Presentation}

A 54-year-old male was referred to our cancer center due to CLL progression. He was diagnosed with CLL 2 years ago, was on active surveillance, but over the last 3 weeks developed excessive fatigue, exertional shortness of breath, and growing lumps in the neck and groin. Past medical history included hypertension, chronic headaches and fatigue, and an excised dermal squamous cell carcinoma. At presentation, he looked unwell, was overweight (BMI 34.9), and had diffused lymphadenopathy and marked splenomegaly. Complete blood count with differential showed normocytic anemia (hemoglobin $72 \mathrm{~g} / \mathrm{L}$ ), thrombocytopenia (platelet count $74 \times 10^{9} / \mathrm{L}$ ), leukocytosis (white blood cell count $135 \times 10^{9} / \mathrm{L}$, mainly due to lymphocytosis [absolute lymphocyte count was $130 \times 10^{9} / \mathrm{L}$ ] as shown in Fig. 1a) and presence of multiple SCs (Fig. 1b). No flow cytometry (FC) was conducted before a consultation at the cancer center. Original complete blood count from $\mathrm{PB}$ reported lymphocytosis with presence of SCs.

Further workup included FC from PB, staging computer tomography (CT), lymph node biopsy, and bone marrow (BM) examination. The CT of the neck, chest, abdomen, and pelvis revealed significant lymphadenopathy (largest nodes range: $2.2-3.2 \mathrm{~cm}$ ) and splenomegaly (16.4 $\mathrm{cm}$ in the longest dimension). FC revealed the presence of the mature monoclonal B-cell population expressing positivity for CD19/20/23/25//79b and FMC7 markers; co-expression of CD5 and a lambda light chain restriction. There were no increases in plasma cells, monocytes, or blasts. BM aspirate was a dry tap, and core biopsy showed a hypercellular marrow with approximately $95 \%$ involvement by B-cells (Fig. 2a). Excisional biopsy of the left neck lymph node showed a monomorphic lymphoid proliferation with a diffuse pattern (Fig. 2b). Most of the cells were small to medium-sized lymphocytes with slightly irregular nuclear contours. On immunohistochemistry, cells were positive for CD20 (Fig. 2c), CD5, Bcl2; negative for CD23, CD3, CD10, and expressed high levels of Cyclin D1 (Fig. 2d).

\section{Karger's}




\section{Case Reports in Oncology}

\begin{tabular}{l}
\hline Case Rep Oncol 2021;14:950-956 \\
\hline DOI: 10.1159/000516748
\end{tabular} \begin{tabular}{l}
$\begin{array}{l}\odot \text { 2021 The Author(s). Published by S. Karger AG, Basel } \\
\text { www.karger.com/cro }\end{array}$ \\
\hline
\end{tabular}

Susman et al.: Approach to Lymphocytosis
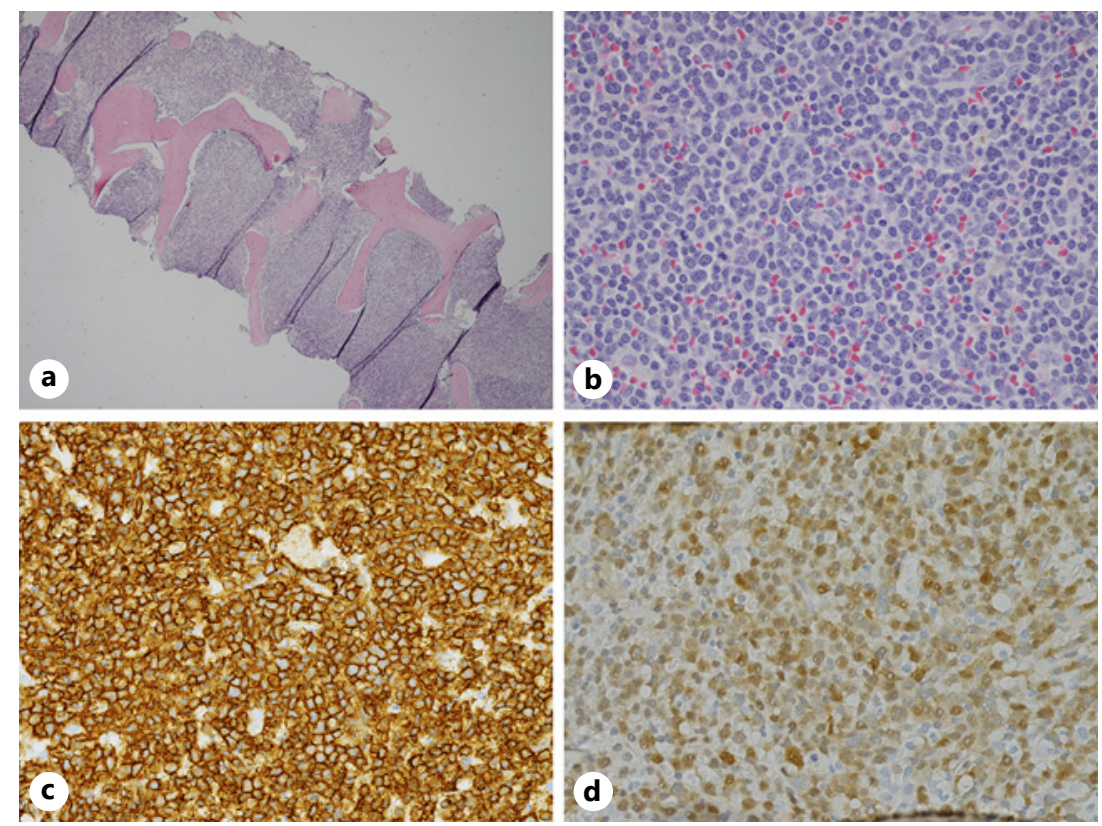

Fig. 2. a HE-stained sections show hypercellular BM due to extensive lymphoid infiltrate, original magnification, $\times 40$. b Lymph node biopsy showing monomorphic lymphoid proliferation with a diffuse pattern, HE staining, original magnification, $\times 400$. c immunohistochemistry from lymph node showing cytoplasmic localization of CD20, original magnification, $\times 400$. d immunohistochemistry from lymph node showing lymphoid cells, expressing high levels of nuclear Cyclin D1, original magnification, $\times 400$. BM, bone marrow.

The Ki-67 proliferative index was 70-80\%. The referring diagnosis CLL was changed to a definitive diagnosis of mantle cell lymphoma, stage IVA with significant BM involvement and leukemic PB. An induction chemoimmunotherapy with intensive supportive care was initiated. The patient received 3 cycles of R-CHOP (rituximab, cyclophosphamide, doxorubicin, vincristine, and prednisone) and 3 cycles of R-DHAP (rituximab, dexamethasone, Ara-C, and cisplatin), followed by consolidative autologous stem cell transplantation and rituximab maintenance. The patient reached complete remission and remained in remission for over 3 years.

\section{Discussion/Conclusion}

CLL is the most common form of adult leukemia in Western countries. The median age at diagnosis is approximately 70 years [2]. It frequently presents with incidental findings of lymphocytosis on routine blood work and is often accompanied by the presence of SCs on a PB smear. Similar findings, however, can result from other benign and malignant conditions that can mimic CLL.

In the case presented above, the initial diagnosis of CLL was determined based on the presence of lymphocytosis and SCs in the PB smear. This resulted in a delay of appropriate investigations, diagnoses, and initiation of therapy. Unlike CLL that has an indolent course with linear progression, MCL's course is moderately aggressive and variable requiring more frequent follow-up and earlier therapy. By the time the patient presented to the cancer center, he was in urgent need to start aggressive chemoimmunotherapy. Due to his BM suppression, he required dose reduction and extensive supportive therapy with multiple blood product transfusions. This may have been avoidable with a more timely MCL diagnosis. The patient 
presented above illustrates this diagnostic uncertainty: CLL was assumed based only on lymphocytosis and SC in the PB without confirmation of a diagnosis by FC.

Approach to Persistent Lymphocytosis in $P B$

Lymphocytosis refers to an increase of a PB lymphocyte count of $>3.0-4.0 \times 10^{9}$ cells $/ \mathrm{L}$ in most laboratories. Lymphocytosis could be primary (malignant, monomorphic, and clonal) due to LPDs or secondary (reactive, pleomorphic, and nonclonal). Viral and bacterial infections are the leading causes of secondary lymphocytosis. Noninfectious causes are attributed to stress, trauma, medications, autoimmune diseases, or inflammation. Detailed patient history and physical examination help to determine the cause of lymphocytosis. Secondary lymphocytosis is usually transient and self-limiting. Persistent lymphocytosis for more than 3 months is more likely associated with an LPD. In addition to a physical examination focusing on lymphadenopathy and/or hepatosplenomegaly, further investigation with FC is essential for diagnosis. FC discriminates clonal versus nonclonal lymphocytosis, identifies cell lineages (B, T, or NK lymphoid cells), and provides an immunophenotype of malignant lymphoid cells based on the presence or absence of specific cell-surface CD antigens.

\section{PB Lymphocytosis Is Not Equivalent to CLL}

Although frequent in appearance, lymphocytosis is not exclusively associated with CLL. PB lymphocytosis also occurs in various B- and T-cell LPDs. Up to $30 \%$ of lymphocytosis may be due to LPDs other than CLL [3]. The characteristics of LPDs presenting with lymphocytosis are summarized in Table $1[2,4]$. While CLL is one of the most common LPDs, additional diagnostic examination of cell morphology and immunophenotyping are required to establish an accurate diagnosis.

\section{SCs in PB Smears Are Not Diagnostic for CLL}

SCs, also known as "Gumprecht cells" or "basket cells," are remnants of leukocytes [5] that lack cytoplasm, and often are presented as smudged nuclei (Fig. 1b). SC can be identified within both reactive and malignant lymphocytosis. SCs are most commonly associated with CLL and are often interpreted as evidence of a CLL diagnosis. Matos et al. [6] demonstrated that SCs had an odds ratio of only 1.97 for discriminating CLL from other LPDs. A more recent review showed frequent presence of SCs in out-of-hospital cardiac arrests, infections, hematological, and solid cancers. Interestingly, the study showed that in all cancers, those patients who had a higher percentage of SCs were prone to have poorer outcomes [7]. Nevertheless, the presence of SCs in PB represents a nonspecific finding that is suggestive but not pathognomonic of CLL.

In conclusion, CLL is often assumed based on the presentation of lymphocytosis with SCs on a PB smear. Although SCs are seen more frequently and in higher quantities in CLL, they are not pathognomonic and can be present in a variety of malignant and nonmalignant conditions. Lymphocyte morphology on PB smear and immunophenotyping by FC should be conducted. Several LPDs present with different degrees of clonal lymphocytosis. FC helps to establish the correct diagnosis of an LPD and determine appropriate further management of the specific disease type.

\section{Statement of Ethics}

The research was conducted ethically in accordance with the World Medical Association Declaration of Helsinki. Written informed consent was obtained from the patient for publication of this case report. The completed consent form is available to the editor if requested. It is a case report, thus the paper is exempt from Ethical Committee approval.

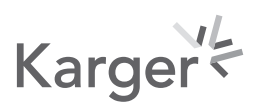


Case Reports in Oncology
Case Rep Oncol 2021;14:950-956

Susman et al.: Approach to Lymphocytosis

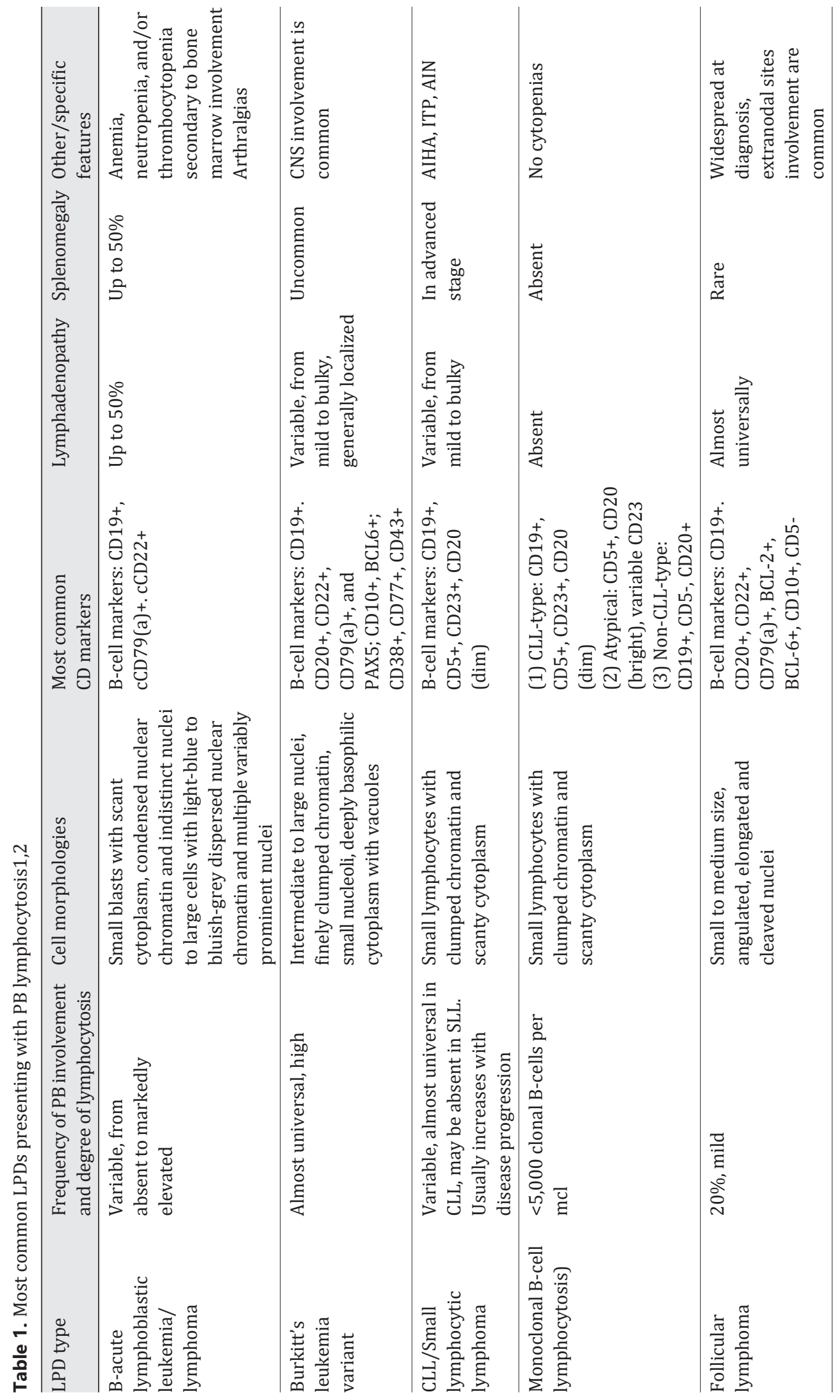




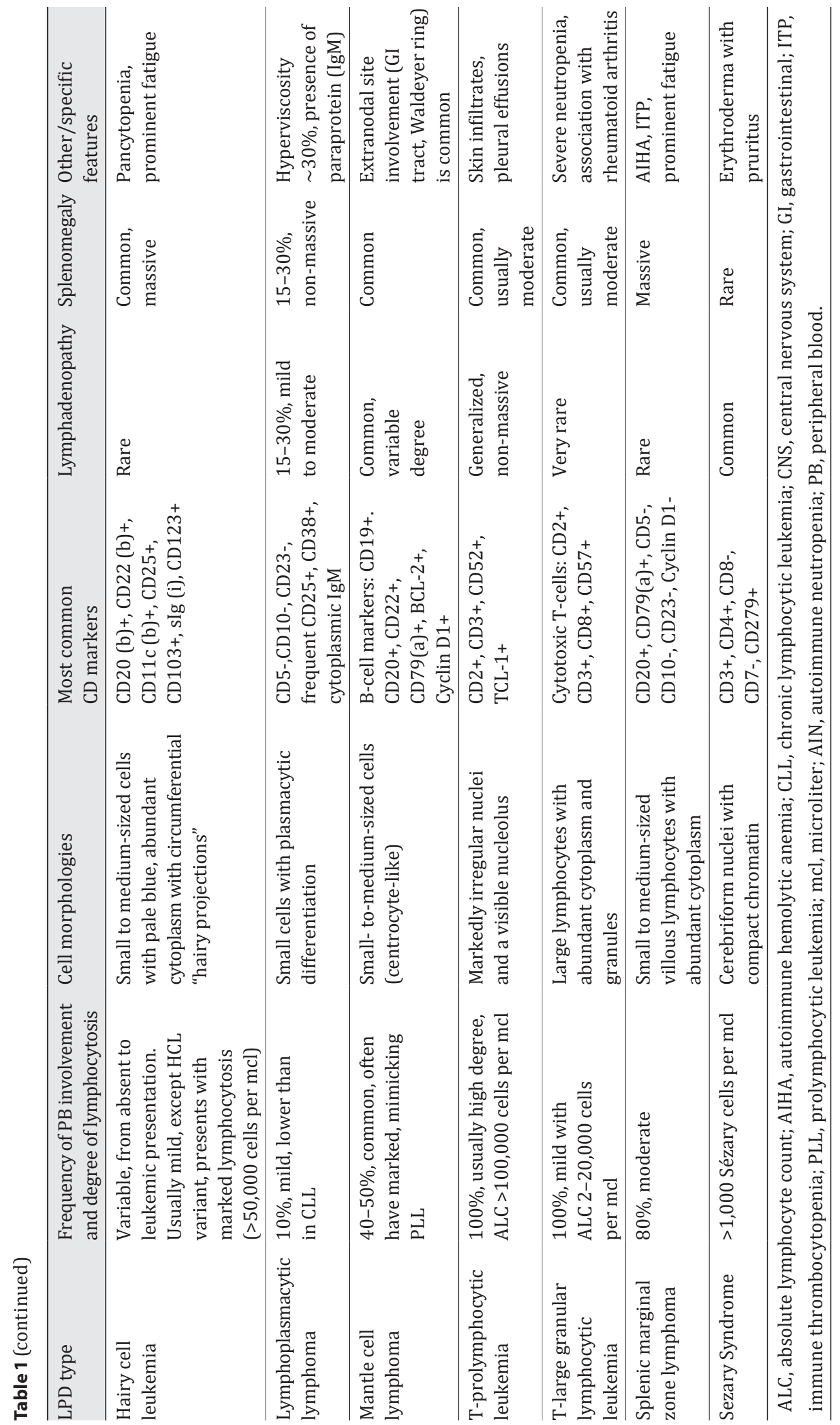




\section{Conflict of Interest Statement}

The authors have no conflicts of interest to declare.

\section{Funding Sources}

No research support for this study.

\section{Author Contributions}

D.S., R.P., and R.K. wrote and reviewed the manuscript. All the authors approved the final version to be published and agreed to act as guarantors of the work.

\section{References}

1 Alberta Health Services. Chronic lymphocytic leukemia: clinical practice guideline LYHE-007 version 6; 2019. https://www.albertahealthservices.ca/assets/info/hp/cancer/if-hp-cancer-guide-lyhe007-cll.pdf.

2 Swerdlow SH, Campo E, Harris NL. World Health Organization classification of tumours of haematopoietic and lymphoid tissues. 4th edn. Lyon: International Agency for Research on Cancer; 2017.

3 Arber DA, George TI. Bone marrow biopsy involvement by non- Hodgkin's lymphoma. Frequency of lymphoma types, patterns, blood involvement and discordance with other sites in 450 specimens. Am J Surg Pathol. 2005; 29(12):1549-57.

4 Kotchetkov R, Price R. Microcellular variant of T-prolymphocytic leukemia mimicking CLL. J Blood Disord Med. 2016;1(1).

5 Binet JL, Baudet S, Mentz F, Chevance A, Lesty C, Blanc C, et al. Basket cells or shadow cells of Gumprecht: a scanning electron microscope study, and the correlation between percentages of basket cells, and cells with altered chromatin structure (dense cells), in chronic lymphocytic leukemia. Blood Cells. 1993;19(3):573-9.

6 Matos DM, Perini G, Kruzich C, Rego EM, Falcão RP. Smudge Cells in peripheral blood smears did not differentiate chronic lymphocytic leukemia from other B-cell chronic lymphoprolipherative diseases. Rev Bras Hematol Hemoter. 2008;31(5):333-6.

7 Chang CC, Sun JT, Liou TH, Kuo CF, Bei CH, Lin SJ, et al. Clinical significance of smudge cells in peripheral blood smears in hematological malignancies and other diseases. Asian Pac J Cancer Prev. 2016;17(4):1847-50. 